\title{
Visual attentional load affects the frequency of involuntary autobiographical memories and their level of meta-awareness
}

\author{
Manila Vannucci $^{1} \cdot$ Claudia Pelagatti $^{1} \cdot$ Maciej Hanczakowski ${ }^{2} \cdot$ Carlo Chiorri $^{3}$ \\ Published online: 6 September 2018 \\ (C) Psychonomic Society, Inc. 2018
}

\begin{abstract}
Involuntary autobiographical memories (IAMs) are memories of past events that come to mind without deliberate retrieval attempts. Common in everyday life, IAMs have recently become a topic of experimental investigations with laboratory procedures. In the present study, we build on the recent methodological advancements in the study of IAMs, and we investigate the effects of manipulating the attentional load on the incidence of IAMs, as well as on the level of meta-awareness of these memories. In two experiments, attentional load was manipulated by varying the demands of the focal vigilance task, and reports of IAMs were collected. In Experiment 1, participants were instructed to stop the vigilance task whenever mental contents unrelated to the task came to their minds (self-caught method). In Experiment 2, participants were intermittently interrupted and probed regarding the contents of their experience (probe-caught method) and the level of meta-awareness for these contents. In both experiments, we found a reduction in the frequency of reported IAMs under increased attentional load. Moreover, in Experiment 2, IAMs were characterized by varied levels of meta-awareness, which was reduced by increased attentional load. These results indicate that allocation of attentional resources toward a focal task reduces reporting of IAMs experienced while performing this task because attentional resources play a role in both retrieval of IAMs and the realization that one is experiencing a memory.
\end{abstract}

Keywords Involuntary autobiographical memories $\cdot$ Autobiographical memory $\cdot$ Meta-awareness $\cdot$ Attentional load

For a long time, research on autobiographical memory has been mainly focused on the investigation of deliberately retrieved memories of personal events, intentionally generated in response to specific cues provided by the experimenter (for a review, see Conway \& Pleydell-Pearce, 2000). However, in many situations in our daily lives we find ourselves being engaged in memories of personal events that come to mind with no conscious or deliberate attempt directed at their retrieval (Berntsen, 1996, 2010; Mace, 2007). For example, striving to attend to a lecture, students may find themselves remembering a party they went to on a previous night. While travelling on a train, reading the words "broken glass" in a

Manila Vannucci

manila.vannucci@psico.unifi.it

1 Department of NEUROFARBA-Section of Psychology, University of Florence, Via San Salvi 12, Padiglione 26, 50135 Florence, Italy

2 SWPS University of Social Sciences and Humanities, Warsaw, Poland

3 Department of Educational Sciences, University of Genoa, Genoa, Italy novel might trigger memory retrieval of a personal episode of stepping on broken glass hidden in the sand and being taken to a hospital. In the past 2 decades, there has been a surge of interest in both psychology and neuroscience toward the investigation of such involuntary autobiographical memories (IAMs; see, for a review, Berntsen, 2010).

Until recently, the most common approach for studying IAMs was the naturalistic diary method, in which individuals are asked to keep a diary of the IAMs they experience in everyday life (e.g., Berntsen, 1996; Berntsen \& Hall, 2004; Mace, 2004). Studies using the diary method established two major features of IAMs as experienced outside the laboratory. First, as in the "broken glass" example reported above, the majority of IAMs are elicited by identifiable external cues, generally related to prominent aspects of the remembered experiences (cue-memory match; e.g., Berntsen, 1996; Berntsen \& Hall, 2004). Second, IAMs are more likely to occur during undemanding activities that require little attention or concentration.

Over the past years, the study of IAMs has been extended to laboratory settings. Building on the first regularity of IAMs - their cue-dependent nature-a number of 
experimental procedures have been developed that aim specifically at eliciting IAMs (e.g., Ball, 2007; Schlagman \& Kvavilashvili, 2008; Vannucci, Batool, Pelagatti, \& Mazzoni, 2014). These procedures, by gaining control over triggers for IAMs, allowed for a detailed examination of the question concerning the properties of the cues that are effective in eliciting IAMs. For example, in the study by Schlagman and Kvavilashvili (2008), participants were asked to perform a vigilance task while being simultaneously exposed to task-irrelevant cue phrases. Participants were instructed to stop the procedure whenever they experienced an IAM, to record basic details about the memory (i.e., memory description, triggers, concentration rating), and then to resume the vigilance task. In the study, the majority of IAMs reported during the task were triggered by the word cues on the screen, with cues of negative emotional valence being more effective in eliciting IAMs compared with positive and neutral cues. Using a modified version of this experimental paradigm, Mazzoni, Vannucci, and Batool (2014) directly compared the effectiveness of verbal and pictorial cues in eliciting IAMs and showed that more IAMs were elicited when verbal cues were presented during a vigilance task. In a related vein, Berntsen, Staugaard, and Sørensen (2013) showed that only cues that uniquely pointed to a single memory, at the exclusion of other memory records, were capable of producing cue-memory matches strong enough to elicit IAMs (see also Rubin, 1995).

While the issue of cue dependence of IAMs received much empirical scrutiny, the second regularity concerning IAMs derived from the diary studies - their preponderance in states of diffused rather than concentrated attention-remains understudied. It is thus the purpose of the present study to examine - with the use of a controlled experimental procedure - whether the attentional requirements of the focal task do indeed modulate the incidence of reported IAMs. Additionally, the present study aims at elucidating the locus of any such effect. Specifically, we are interested whether changes in the incidence in reporting of IAMs may result from changes in how often IAMs are retrieved or changes in participants' ability to realize that they experience an IAM, which is another precondition for reporting memories that have been spontaneously retrieved in response to environmental triggers.

The results of diary studies suggest that the frequency of IAMs may depend on the attentional demands of the ongoing task, but the intrinsic limitations of such studies - the inability to manipulate variables being the most obvious pitfall- prevent the possibility of identifying the exact mechanism (s) by which attentional load influences the occurrence of IAMs. To our knowledge, the role of attention in the elicitation of IAMs has been experimentally investigated only in two studies. Ball (2007) had participants produce free associations to word cues (concrete nouns). At the end of the trial, participants were asked to report if a personal experience had come to their minds while they were giving the free-association responses. Importantly, while providing free associations, half of the participants (control condition) observed an unchanging box in the middle of the computer screen, whereas the other half (dual-task condition) were asked to perform a secondary task of pressing a button in response to a color-changing box. The comparison between the two conditions revealed that more associates were produced before an IAM was reported when attentional load was high, suggesting that attentional load may negatively impact upon spontaneous retrieval of IAMs. Similarly, Barzykowski and Niedźwieńska (2018) used a divided attention manipulation in a procedure for investigating IAMs in the laboratory (see Schlagman \& Kvavilashvili, 2008) and observed reduced incidence of IAMs under divided attention conditions, once again suggesting that stronger engagement of attention interferes with reporting of IAMs.

The results obtained by Ball (2007), as well as Barzykowski and Niedźwieńska (2018), converge with the results of diary studies in suggesting the role of attentional load in modulating IAMs. However, the specific mechanism by which this modulation occurs remains unclear. Specifically, we argue that the reduction in the rate of IAMs under high levels of attentional load might arise for two reasons. First, attention-demanding activities might hamper the involuntary retrieval of autobiographical memories. This may occur because attention allocated to a demanding focal task is removed from retrieval-related processes such as developing a memory (Baddeley, 1993) and/or inhibiting the memories competing for retrieval access (see Anderson \& Spellman, 1995; Mandler, 1994). Also, as described earlier, research suggests that most of the IAMs are triggered by identifiable cues in the environment. Increasing attentional load may reduce processing of environmental stimuli that are not immediately pertinent to the focal task, consistently with the number of studies showing that additional attentional load reduces processing of distraction (see Sörqvist \& Marsh, 2015, for a review). Thus, attentional load may reduce the incidence of IAMs because it either impacts on the core process of retrieval or creates conditions under which retrieval is not triggered due to insufficient processing of environmental cues.

Second, apart from these memory effects, where retrieval of IAMs becomes disrupted, a postretrieval effect of increased attentional load also seems possible. Specifically, attentiondemanding activities might impact upon higher monitoring processes that determine whether retrieved IAMs become reported. Koriat and Goldsmith (1996) have argued that when considering output of memories, a number of postretrieval processes should be taken into account, particularly if decisions whether to volunteer a memory or withhold it remain under the person's control (see also Goldsmith, Koriat, \& Weinberg-Eliezer, 2002; Hanczakowski, Pasek, Zawadzka, \& Mazzoni, 2013). When a specific memory question is asked, a person monitors the accuracy of information retrieved 
from memory to decide whether this information meets the criteria necessary for volunteering it as a response to the given question. In the case of IAMs arising spontaneously-without any specific memory query - such monitoring of accuracy does not occur, but it does not mean that retrieved information is not monitored. The literature on mind wandering stresses that all contents of the mind are to some extent subjected to monitoring processes referred to as meta-awareness: a graded realization of the contents of one's mind (see Schooler, 2002; Schooler et al., 2011, for a discussion). Thus, even in the absence of specific requirements contained in a memory query, the contents of mind are subjected to monitoring that determines whether one is conscious that a memory has been retrieved. We postulate that such a monitoring process is a constant process accompanying any cognitive endeavor, but the effectiveness of such monitoring and thus its output — a specific state of meta-awareness of the contents of one's mind in a given moment - can be determined by multiple factors, such as an explicit requirement to report on the contents of one's mind in the experimental procedure, the features of the mental contents (e.g., one is perhaps more likely to realize that a memory is experienced if this memory is traumatic; but see Takarangi, Strange, \& Lindsay, 2014, for the evidence that people may lack meta-awareness of trauma-related thoughts) or the availability of attentional resources. Just as described by Koriat and Goldsmith (1996), monitoring is likely to determine whether a memory would be volunteered, as without realization that one is remembering, retrieved information cannot be reported as IAM. If attentional load were to affect such monitoring of the contents of consciousness in the form of meta-awareness, then reduced incidence of IAMs may reflect not only reduced memory access but also reduced realization that memories have been retrieved.

In the context of research on autobiographical memory, a recent study by Vannucci et al. (2014) found that people do not always notice that they have had an IAM during a vigilance task, and they might then omit reporting such memories on numerous occasions. Using the methodology developed in studies on mind wandering (see Smallwood \& Schooler, 2015, for a review), Vannucci et al. compared the preponderance of IAMs when participants were asked to report them whenever they occurred (the so called self-caught method) and were interrupted and asked about their experience (the so called probe-caught method or experience sampling). If a person does not always realize that an IAM has been retrieved - in other words, if there are gradations in the output of monitoring process in the form of meta-awareness - then more IAMs should be observed for the probe-caught method than for the self-caught method. This should occur because meta-awareness (or rather the lack of it) plays no role in reporting the contents of the mind in the probe-caught method in which high levels of monitoring are imposed by a specific question about the contents of one's mind. This pattern of greater incidence of IAMs in the probe-caught rather than self-caught method was indeed what Vannucci et al. observed, substantiating the claim that IAMs can differ in the degree to which they are accompanied by meta-awareness - a potential locus of attentional load effects examined in the present study. In addition to that, recent studies on trauma-related intrusions reported that people often failed to recognize the occurrence of intrusive thoughts, suggesting that people may lack metaawareness of their trauma-related thoughts (Takarangi et al., 2014; Takarangi, Lindsay, \& Strange, 2015; Takarangi, Nayda, Strange, \& Nixon, 2017).

Overall, the aims of the present study were (1) to examine the role of attentional load in reporting IAMs, and (2) to elucidate the mechanisms by which attentional load affects reporting of IAMs, assessing separately the effects on both retrieval and postretrieval (meta-awareness) processes. In two experiments, we manipulated attentional load associated with the focal task in a between-subjects design, with high attentional load being experienced by one group (High-AL), and low attentional load by the other (Low-AL). To assess IAMs, we employed a modified version of the vigilance task with irrelevant cue-words developed by Schlagman and Kvavilashvili (2008), already used in previous studies of IAMs (Barzykowski \& Niedźwieńska, 2016; Mazzoni et al., 2014; Vannucci et al., 2014; Vannucci et al., 2015). In Experiment 1, IAMs were assessed using the self-caught method, by which participants were instructed to stop the vigilance task whenever mental contents unrelated to the task came to their minds. The reported contents were further analyzed to extract instances of IAMs. The purpose of Experiment 1 was to establish the basic pattern of how changes in attentional load affect reporting of IAMs. We predicted that increased attentional load would reduce the incidence of reported IAMs, and this pattern has indeed been observed.

In Experiment 2, we applied the same experimental manipulation as in Experiment 1, but we assessed IAMs with the probe-caught method, by which participants were intermittently and pseudorandomly interrupted and probed regarding the contents of their experience. By using the probe-caught method, the role of monitoring in reporting IAMs should be minimized, allowing for a clearer examination of the retrieval dynamics. Moreover, the probes used in Experiment 2 were also accompanied by a meta-awareness rating, asking how aware participants were of where their attention was focused immediately prior to the probe. In this way, we were able to assess the level of meta-awareness associated with retrieved IAMs in different attentional load conditions (see also Poh, Chong, \& Chee, 2016). If attentional load reduces the incidence of IAMs by interfering with their retrieval, we expect to document here the same pattern with the probe-caught method as with self-caught method used in Experiment 1, namely, a reduction in the number of IAMs reported under high attentional load condition. If attentional load reduces participants' 
ability to realize that they experience IAMs, we expect to document reduced levels of meta-awareness in Experiment 2.

In both experiments phenomenological properties of IAMs, such as specificity, vividness, pleasantness, and intensity of the feeling experienced during retrieval, were also assessed and analyzed for exploratory purposes. It was investigated whether any effect of attentional load on reporting of IAMs is also accompanied by the changes in how these IAMs are experienced, over and above the issue of whether people realize that these memories have been retrieved.

\section{Experiment 1}

\section{Method}

\section{Design}

The experiments in this study conformed to a betweensubjects design comparing the effect of attentional load (low vs. high) on the number of IAMs and their phenomenological properties. For those IAMs that were reported by participants as being triggered by cues presented on the screen, retrieval times were also collected and analyzed.

As no previous research has addressed a similar issue, we had no a priori information to determine an expected effect size. Hence, given the often poor replicability of effects of small and moderate size, we decided to test a number of participants that could allow us to detect significant differences with a large effect size. It has been suggested that this approach can help increase the replicability of results of psychological studies (Asendorpf et al., 2013), provided that one takes care to check for irreproducible outliers that may lead to "fluke" findings, given the relatively smaller sample size (Funder et al., 2014). Since we planned to mainly perform independent-samples $t$ tests, we computed through G*Power 3.1.7 (Faul, Erdfelder, Lang, \& Buchner, 2007) that, in order to detect significant differences with a large effect size ( $d$ $\geq 0.80$ ) with a significance level (two-tailed) of .05, a statistical power of.80, and the same number of participants in each group, a minimum total sample size of 52 participants could be considered adequate. Since some participants could drop out from the experiment at any time and/or problems with the data collection devices could lead to missing or invalid data, slightly more participants were recruited.

\section{Participants}

Sixty-four undergraduate students from the University of Florence ( 42 females, mean age $=21$ years, $S D=2.6$ years; age range: $18-35$ years) were randomly assigned to one of the two conditions, High-AL $(n=32)$ and Low-AL $(n=32)$. They were all native Italian speakers, with normal or corrected-to- normal vision. Groups did not significantly differ in age and sex ratio.

\section{Materials}

Vigilance task During the experimental session, participants completed a modified version of the vigilance task developed by Schlagman and Kvavilashvili (2008) and used in previous studies to investigate IAMs (Mazzoni et al., 2014; Vannucci et al., 2014; Vannucci, Pelagatti, Chiorri, \& Mazzoni, 2016; Vannucci et al., 2015). The task consisted of 510 trials of target detection, presented in a pseudorandom order (see below), each remaining on the screen for $1.5 \mathrm{sec}$. In the Low-AL condition, on each trial, an image was shown on the computer screen depicting either a pattern of black horizontal and black vertical lines (nontarget stimuli) or a pattern of black horizontal and red vertical lines (target stimuli). In the High-AL condition, nontarget stimuli consisted of a pattern of red horizontal and black vertical lines, whereas the target consisted of a pattern of black horizontal and red vertical lines (as in the Low-AL condition; see Fig. 1).

In both groups, the target stimuli appeared on 10 trials, and they were presented pseudorandomly, that is, every 40-60 trials, in order to ensure that they occurred at long and irregular intervals (Schlagman \& Kvavilashvili, 2008; Vannucci et al., 2014; Vannucci et al., 2015). Word phrases that were to serve as cues for IAMs (e.g., "washing hands", "favorite food", "falling down stairs") were placed in the middle of the image, presented in 18-CPI Arial font, and displayed without obscuring any of the lines. These were included on 102 trials (one fifth of the total number of trials). The word phrases were selected from the Italian adaptation of a standardized pool of 800 word phrases developed by Schlagman and Kvavilashvili (2008) and already used in previous studies on IAMs (for more details on the adaptation, see Vannucci et al., 2015). Equal numbers of neutral $(n=34)$, positive $(n=34)$, and negative $(n=34)$ cues were presented during the task.

Memory characteristics questionnaire Participants recorded details of their memories on a modified version of a questionnaire used in previous studies on IAMs (Vannucci et al., 2015). We asked participants to rate the vividness of the memory (from $1=$ very vague, almost no image at all to $5=$ very vivid, almost like normal vision), its pleasantness ( $1=$ very unpleasant; 3 = neutral; 5 = very pleasant), and the intensity of the feeling experienced at retrieval $(1=$ none; $5=a$ lot $)$. We also asked participants to specify whether the remembered event was general or specific. Participants received instructions on how to identify a general and a specific memory (for more details, see Vannucci et al., 2016). At the end of the experiment, participants also rated their overall level of concentration $(1=$ not at all concentrated; $5=$ fully 


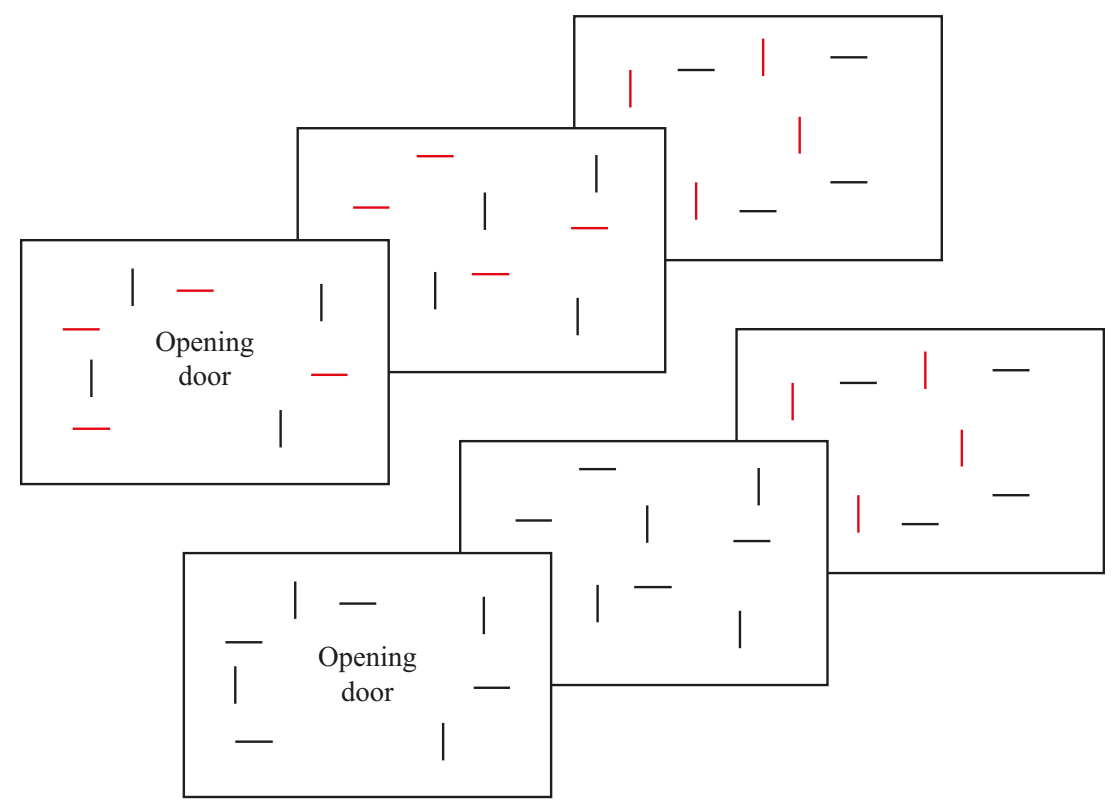

Fig. 1 Example of the stimulus displays in high attentional load (High-AL; top) and low attentional load (Low-AL; bottom) group

concentrated) and the level of boredom experienced during the task $(1=$ not at all; $5=$ very bored $)$.

\section{Procedure}

Participants were tested individually. After completing the informed consent form, participants were told that they would take part in a study examining concentration using a vigilance task, and they were instructed to detect target stimuli (images with red vertical lines and black horizontal lines) among a large number of nontarget stimuli by saying "yes" out loud each time they detected a target stimulus. They were told that they would also see short word phrases in some of the trials, but they were not supposed to do anything with these items. It was explained that the condition they were taking part in was looking at how people could keep their concentration on the patterns (line-detection group) and that in another condition (word-detection group) participants would have to concentrate on the words (this was a cover story, the word-detection group did not exist). Participants were further instructed that, due to the task being quite monotonous, they could find themselves thinking about other things, which was quite normal. They were told that if any task-unrelated mental content (mental contents could refer to thoughts, intentions, plans for the future, past experiences, etc.) crossed their mind during the task, they should click the mouse to interrupt the presentation and write on a sheet of paper a short sentence describing their mental content. They were informed that this initial brief description of the mental content should be sufficient to remind them of that specific mental content at a later point in time. They were also asked to indicate whether the mental content was triggered by something or whether there was no trigger (selecting an option among the following: no trigger, internal thoughts, an element in the environment, a word-phrase on the screen). If the mental content was triggered by a word-phrase shown on the screen, they were asked to write down which one.

After the instructions, participants were given a short 15trials training identical to the experimental task, to practice (i.e., familiarize themselves with the task and the stimuli). Three word phrases were presented during the training phase. At the end of the vigilance task, after all stimuli had been presented and all contents recorded, participants were informed about the nature of involuntary memories and they were presented with the descriptions of their mental contents one by one and asked to indicate the involuntary memories. For each of the involuntary memories, they were asked to complete a brief questionnaire assessing phenomenological properties of memories (see Materials). The session lasted approximately 45 to 60 minutes.

\section{Results and discussion}

We first assessed whether the manipulation of attentional load was effective in varying the demands of the vigilance task by examining the rate of errors in detection of the target perceptual patterns. Indeed, the High-AL and Low-AL groups differed in this respect, $\chi^{2}(1, n=64)=5.38, p=.020, r=.29$, with the participants in the High-AL being more likely to make at least one error (94\%) compared with the Low-AL group $(72 \%)$. We decided to dichotomize the number of errors because $53(82.8 \%)$ participants did not make any error, six (9.4\%) made one error, four (6.3\%) made two errors, and one $(1.6 \%)$ made three errors. 
No significant differences between the two groups were found in the self-reported level of boredom (High-AL: $M=$ 3.00, $S D=0.84$; Low-AL: $M=3.03, S D=1.09), t(62)=$ $-0.13, p=.898, d=0.03$, and concentration (High-AL: $M=$ 3.59, $S D=0.56$; Low-AL: $M=3.41, S D=0.80), t(62)=1.09$, $p=.281, d=0.26$, experienced during the task.

During the vigilance task, participants were asked to report all task-unrelated mental contents that came into their mind. At the end of the task, participants performed the classification of mental contents as memories versus non-memories. Before conducting the data analyses, all mental contents classified as involuntary memories were read through by the experimenter to check that they were autobiographical in nature (semantic "mind-pops" were excluded).

Participants generated a total of 325 involuntary nonmemory contents with a mean of $5.08(S D=3.76$, range: $0-17)$ and 219 IAMs with a mean of $3.42(S D=2.38$, range: $0-9)$ per participant. The majority of reported IAMs $(96.35 \%)$ had an identifiable trigger. Of these, $82.94 \%$ were reported to be triggered by the cues on the screen, $16.11 \%$ by internal thoughts, and $0.95 \%$ by other environmental cues. The comparison of the total number of IAMs between the two groups revealed that the Low-AL group reported more than twice the number of IAMs reported by the High-AL group (Low-AL: $M$ $=4.81, S D=2.07$; High-AL: $M=2.03, S D=1.79), t(62)=$ $5.75, p<.001, d=1.44$. A similar pattern was obtained when the analyses were limited to the subset of IAMs reported as being triggered by the specific cues on the screen: Low-AL reported a higher number of IAMs triggered by the cues compared with High-AL (Low-AL: $M=3.81, S D=1.93$; HighAL: $M=1.66, S D=1.72), t(62)=4.73, p<.001, d=1.18$. Thus, the present results demonstrate how increased attentional load reduces the incidence of IAMs

We also compared in the two groups the mean proportion of memories that were reported to have a trigger. Triggers could be a cue, a thought or an environmental stimulus. Because environmental cues were reported as triggers of a memory in only two cases, they were not further analyzed. There were no significant differences between High-AL and Low-AL in the mean proportion of IAMs triggered by the cues (High-AL: $M=0.75, S D=0.34$; Low-AL: $M=0.78$, $S D=0.25), t(55)=0.34, p=.733, d=0.10$, or by thoughts (High-AL: $M=0.22, S D=0.34$; Low-AL: $M=0.17, S D=$ $0.23), t(55)=-0.67, p=.504, d=0.18$.

Next, we assessed whether the experimental manipulation affected the phenomenological qualities of IAMs. In this case the phenomenological qualities were rated for each reported IAM and could vary not only between participants but also within participants. Hence, we had to consider as a unit of analysis a single memory. Given that participants could report more than one IAM, we used a multilevel (or hierarchical) data set, in which IAMs were nested into participants. The use of this strategy of analysis not only allowed us to take into account the nonindependence of the units of analysis, but also to accommodate unequal numbers of data points within participants (Jahng, Wood, \& Trull, 2008). We thus specified random-intercept multilevel models to test for associations of the group factor (Low-AL vs. High-AL) with the ratings of phenomenological qualities of the IAMs, which were considered as the dependent variables. No significant differences between the two groups were found in any phenomenological qualities of IAMs (see Table 1), nor in their specificity (odds ratio with "Low" as reference: $0.88, \mathrm{CI}[0.43,1.74], p=.746$ ). These results suggest that the characteristics of IAMs are largely unaffected by the way attentional load has an effect on the process of creating them.

For those IAMs that participants reported as being triggered by word phrases shown on the screen, retrieval times (RTs, as in Schlagman \& Kvavilashvili, 2008) were calculated. RTs were calculated by adding the RT for the present (clicked on) trial, to the RTs for all the trials back, up to the trial that presented the word that was reported by the participant as the trigger of the mental content. Similarly to the analyses on the phenomenological qualities of the IAMs, we specified random-intercept multilevel models. Given that RTs in this experiment were substantially skewed (4.53) and kurtotic (29.77), we conducted the analysis of retrieval times of IAMs after log transformation of RTs. The analysis revealed an effect of the group, $F(1,59.30)=5.59, p=.021$, where RTs were slower in the High-AL group (estimated $M=$ $3.84,95 \%$ CI $[3.73,3.94])$ compared with the Low-AL group (estimated $M=3.68,95 \%$ CI $[3.61,3.76]$ ). Thus, not only increased attentional load has reduced the incidence of IAMs, as discussed earlier, but it also increased the time it took participants to report such IAMs.

Table 1. Phenomenological qualities of involuntary autobiographical memories in High-Attentional Load (High-AL) and Low-Attentional Load (Low-AL) groups in Experiments 1 and 2

\begin{tabular}{llllll}
\hline Variable & \multicolumn{2}{l}{ Estimated group means $(95 \% \mathrm{CI})$} & $\mathrm{F}$ & $\mathrm{df}$ & $\mathrm{p}$ \\
\cline { 2 - 3 } & & & & \\
Low-AL High-AL & & & & \\
\hline
\end{tabular}

\begin{tabular}{lllllll}
\hline Experiment 1 & & & & & \\
$\mathrm{n}$ & 32 & 32 & & & \\
Vividness & $4.20(4.01-4.38)$ & $4.10(3.84-4.36)$ & 0.34 & $1,60.54$ & .559 \\
Pleasantness & $3.35(3.11-3.59)$ & $3.29(2.94-3.64)$ & 0.07 & $1,64.92$ & .795 \\
Intensity & $3.36(3.13-3.60)$ & $3.18(2.85-3.51)$ & 0.80 & $1,64.41$ & .374 \\
Experiment 2 & & & & & \\
$\mathrm{n}$ & 29 & 29 & & & \\
Vividness & $3.99(3.69-4.28)$ & $3.92(3.54-4.31)$ & 0.06 & $1,51.13$ & .793 \\
Pleasantness & $2.95(2.64-3.25)$ & $3.12(2.70-3.54)$ & 0.46 & $1,44.15$ & .498 \\
Intensity & $3.30(2.98-3.62)$ & $3.05(2.63-3.47)$ & 0.91 & $1,40.28$ & .345 \\
\end{tabular}

Note: CI: confidence interval; $\mathrm{df}=$ degrees of freedom; $\mathrm{n}=$ number of participants 


\section{Experiment 2}

The main finding of Experiment 1 is that increased attentional load reduces the incidence of self-reported IAMs. However, such a reduction may arise for two reasons: Either IAMs were not retrieved when attentional load was increased, or, alternatively, they were retrieved, but due to deficient monitoring under increased attentional load participants failed to realize that they were experiencing IAMs and consequently did not stop the experimental procedure to report them. In other words, the attentional load manipulation may have interfered with the retrieval processes or it may have reduced the ability to accurately monitor one's mind in order to notice and report IAMs. One should also note that these two possibilities are not mutually exclusive, and the effects of attentional load on the incidence of reported IAMs may actually have two loci. In order to separate these two potential mechanisms, in Experiment 2 we assessed IAMs by using a probe-caught method, forcing our participants to scrutinize the contents of their minds and thus minimizing the role of any differences in spontaneous monitoring across experimental conditions. The use of the probe-caught method thus allows for assessing the impact of the attentional load manipulation on retrieval of IAMs. We also supplemented the probes with an additional query explicitly asking participants to assess the level of metaawareness of the mental contents reported in response to the probes. The examination of these assessments allows for assessing the impact of the attentional load manipulation on meta-awareness of IAMs.

\section{Method}

\section{Participants}

Sixty undergraduate students from the University of Florence (47 females, mean age $=21.35$ years, $S D=1.87$ years; age range: 19-29 years) were randomly assigned to one of the two conditions, High-AL $(n=30)$ and Low-AL $(n=30)$. All participants were native Italian speakers, with normal or corrected-to-normal vision.

\section{Materials}

Vigilance task The vigilance task and the experimental manipulation of focused attention were the same as in Experiment 1, but the number of trials was extended from 510 to 800; this was done to increase the number of IAMs collected in the experiment. The target stimuli (black horizontal lines and red vertical lines) appeared on 16 trials and the word phrases on 160 trials (54 were neutral, 53 positive, and 53 negative). At 15 fixed points during the presentation, the vigilance task was stopped and two questions (a probe trial) appeared on the screen. The first question was, "What were you thinking about just immediately prior to the probe?" Participants answered this question on a sheet of paper, selecting one of the following options: "I was focused on the task"; "I was thinking about ..." (with instructions to write down a short sentence describing their mental content and the trigger, if any); and "My mind was blank." The second question presented on the screen was, "How aware were you of where your attention was focused?", and participants indicated their level of awareness by using a 7 -point scale $(1=$ fully aware; $7=$ fully unaware). The answer was reported on the sheet of paper. The first probe was at trial 35 , and there were a minimum of 35 and a maximum of 72 trials between each probe trial.

Memory characteristics questionnaire Participants were asked to fill out the same memory questionnaire used in Experiment 1.

\section{Procedure}

Participants were tested individually in the same way as in Experiment 1 . They received the same training phase and the same tasks upon the completion of the main vigilance sections of the experiments. The instructions were modified to inform participants that they would be interrupted during the performance and presented with thought probes consisting of two questions.

\section{Results and discussion}

Due to a technical error, the data of two participants (one in the High-AL group) were not recorded. The High-AL group was more likely $(93 \%)$ to commit at least one error in the vigilance task than the Low-AL group $(72 \%), \chi^{2}(1, n=58)=4.35, p=$ $.040, r=.27$, confirming that our manipulation served to vary the demands of the focal task. We again decided to dichotomize the number of errors because 48 (82.8\%) participants did not make any errors, seven $(12.1 \%)$ made one error, one (1.7\%) made two errors, one (1.7\%) made four errors, and one $(1.7 \%)$ made six.

No significant differences between the two groups were found in the self-reported level of boredom (High-AL: $M=$ $3.03, S D=0.91$; Low-AL: $M=3.07, S D=0.92), t(56)=0.14$, $p=.886, d=0.04$, and concentration experienced during the task (High-AL: $M=3.38, S D=0.68$; Low-AL: $M=3.59, S D$ $=0.87), t(56)=1.01, p=.315, d=0.27$.

Participants generated a total of 315 involuntary nonmemory contents with a mean of $5.43(S D=2.93$, range: $0-13$ ) per participant and 143 IAMs with a mean of 2.47 (SD $=2.04$, range: $0-9$ ) per participant. The majority of IAMs $(95.10 \%)$ had an identifiable trigger. Of these, $77.21 \%$ were reported to be triggered by the cues on the screen, $16.91 \%$ by internal thoughts, and $2.94 \%$ by other environmental cues. 
The comparison of the total number of IAMs between the two groups showed that the Low-AL group reported significantly more IAMs than did the High-AL group (Low-AL: $M=$ $3.31, S D=2.25$; High-AL: $M=1.62, S D=1.37), t(56)=3.45$, $p=.001, d=0.91$. A similar pattern was obtained when the analyses were limited to the subset of IAMs reported as being triggered by the specific cues on the screen: Low-AL reported a higher number of IAMs triggered by the cues compared with High-AL (Low-AL: $M=2.72, S D=2.03$; High-AL: $M=0.93$, $S D=1.05), t(55)=4.17, p<.001, d=1.10$. These results replicate the main results of Experiment 1, but this time with a probe-catching rather than a self-catching method. The fact that the incidence of IAMs was reduced under high attentional load even when the probe-caught method was used indicates that additional attentional load interferes with retrieval of IAMs, independently of any possible effect on monitoring of mental contents.

We also compared in the two groups the mean proportion of memories that were reported to have a trigger. Triggers could be a cue, a thought, or an environmental stimulus. Environmental cues as triggers of a memory were reported in only two cases and were not further analyzed. Participants in the High-AL group reported a lower proportion of IAMs triggered by the cues compared with Low-AL (High-AL: $M=$ $0.55, S D=0.43$; Low-AL: $M=0.84, S D=0.25), t(51)=3.12$, $p=.003, d=0.85$, but no significant difference was found in the mean proportion of IAMs triggered by thoughts (HighAL: $M=0.16, S D=0.29$; Low-AL: $M=0.09, S D=0.16$ ), $t(47)=-1.23, p=.225, d=0.36$.

Next, we assessed whether the experimental manipulation affected the level of awareness associated with the retrieval of IAMs. As in Experiment 1, we allowed a multilevel structure to the data, since the level of awareness of an IAM could vary both between and within participants. Again, the unit of analysis was the single IAM. Low-AL participants reported a higher level of awareness (lower rating) compared with High-AL (Low-AL: estimated $M=2.63$, 95\% CI [2.08, 3.18]; High-AL: estimated $M=3.63$, 95\% CI [2.97, 4.28]), $F(1,49.40)=5.47, p=.023$. Thus, increased attentional load not only reduced the number of IAMs caught by the probes, as seen in the earlier analysis, but it also reduced awareness of the remaining IAMs that were eventually caught by the probes. These results point to a double-whammy effect of attentional load on IAMs, with the effects located both at the stage of memory retrieval and at the stage of conscious experience of retrieved memories. No significant differences between the two groups were found in any phenomenological qualities of IAMs (see Table 1), nor in their specificity (odds ratio with "Low" as reference: $0.92, \mathrm{CI}[0.29,2.95], p=.885)$. These findings are in line with the results of Experiment 1, showing that the effects of attentional load on the number of reported IAMs are not accompanied by changes in how these IAMs are experienced.

\section{General discussion}

The present study looked at the incidence of IAMs elicited in a laboratory procedure as a function of attentional load of the primary task during which IAMs were collected. Overall, increased attentional load was associated with the reduced number of IAMs, as assessed with both the self-caught (Experiment 1) and probe-caught (Experiment 2) methods. These results indicate that attentional load posed by the focal task in which one is engaged is a major factor determining whether spontaneous and unrelated memories of one's past are likely to occur. The fact that this reduction was observed with the probe-caught method - a procedure specifically designed to equate the postretrieval monitoring processes when reporting an IAM - indicates that one mechanism by which increased attentional load reduces the incidence of IAMs is by limiting retrieval operating within the system of autobiographical memory. However, additional results from Experiment 2 indicate also that increased attentional load not only interferes with the retrieval of IAMs but at the same time reduces the self-reported meta-awareness of the retrieved spontaneous memories. Thus, even when participants realize they experienced IAMs when explicitly asked by the experimenter, they also report that before the question was asked they were less metacognitively aware of their own memories when attentional demands of the focal task were high. Taken together, these results point to a double role of attentional resources in shaping both the retrieval (occurrence of IAMs) and postretrieval processes (meta-awareness of IAMs) related to spontaneously arising autobiographical memories.

By emphasizing the role of attentional load in the occurrence of spontaneous autobiographical memories, our findings are in agreement with the results of the diary studies, which showed that IAMs are more likely to be reported when one is engaged in undemanding activities that require little attention and concentration (Berntsen \& Hall, 2004; Kvavilashvili \& Mandler, 2004). They also join the empirical contribution of Ball (2007), who found that IAMs came to mind later during a word-association task if attentional load was increased under dual-task conditions. Importantly, in the present study, we could clearly demonstrate a causal role of focused attention in controlling IAMs by using a direct experimental manipulation targeting attentional load while equating experimental conditions in terms of other variables such as the amount of visual stimulation and response demands. One should also note, however, that the present study compared only two conditions of attentional load, with a very undemanding task in the Low-AL condition contrasted with a moderately difficult task in the High-AL condition, which could still be completed with a very low error rate. This manipulation was designed to mimic a distinction between tedious, boring tasks and moderately engaging tasks, which has been discussed with reference to diary studies on IAMs (e.g., Berntsen \& Hall, 2004; 
Kvavilashvili \& Mandler, 2004). It is particularly worth noting that the present experiments did not include a condition in which task difficulty would be further increased, possibly limiting participants' engagement in the focal task due to motivational considerations. One should thus not necessarily interpret the present results as indicating that task difficulty per se reduces the incidence of IAMs but rather that task engagement plays such a moderating role, while keeping in mind that the relationship between task difficulty and task engagement need not be straightforward.

The crucial insight that our use of the laboratory procedure enabled was a clear delineation of the double-mechanism account by which attentional load operates both at retrieval and postretrieval levels of cognitive processing within the system of autobiographical memory. The double-mechanism account postulates that increased attentional load reduces the number of reported IAMs because not only does such a load affect retrieval but it also reduces the extent to which people realize what the contents of their minds are. It is worth noting, however, that this formulation clearly requires additional theoretical and empirical work. With regard to retrieval of IAMs, it is not yet clear what stage of the memory process becomes disrupted by the increased attentional load. Retrieval is not a single process but rather a collection of processes which, apart from the core process of accessing memory with the use of cues, includes other processes such as the adoption of a retrieval mode (Evans, Williams, \& Wilding, 2015) and retrieval orientation (Herron \& Rugg, 2003; Zawadzka, Hanczakowski, \& Wilding, 2017) or cue encoding and elaboration (Herron, Evans, \& Wilding, 2016). Some of these subprocesses of retrieval become irrelevant when the focus is on spontaneous cognition; for example, retrieval mode is not required in nonmemory tasks in which IAMs are collected. Still, it is far from clear whether attentional load affects the core processes of accessing memory, particularly in the face of evidence showing relative insensitivity of retrieval to manipulations of cognitive load (Craik, Govoni, Naveh-Benjamin, \& Anderson, 1996). It is possible that increased attentional load reduces the capacity for processing environmental cues (Sörqvist \& Marsh, 2015), which often serve as triggers for IAMs. This possibility requires further investigation in which encoding of cues could be examined independently from retrieval of IAMs.

With regard to postretrieval processes of monitoring the products of retrieval, it is clear that the concept of meta-awareness, as applied to IAMs, should become more closely linked to other forms of memory monitoring. As already signaled earlier, research on deliberate memory elicited by specific memory queries has, for a long time, recognized that products of retrieval are subjected to various forms of monitoring, which determine responses provided in memory reports. Thus, when a specific memory question is asked, the accuracy of information retrieved from memory is monitored. The output of this monitoring determines whether the retrieved information will be volunteered as an answer (Koriat \& Goldsmith, 1996). When asked about phenomenological qualities of retrieved information, people can decide whether it is associated with much contextual information, warranting the report of "recollecting," or whether lacking such contextual information retrieval can be described merely as "knowing" (Wixted \& Mickes, 2010). These forms of monitoring of retrieved information are described in reference to situations in which people are asked memory questions and what they focus on are memory qualities. The argument presented here is that there are also other forms of monitoring - applicable to memories but also all other types of mental contents, as described in the literature on mind wandering (see for a review, Schooler et al., 2011) - which determine not how retrieved information is appraised but whether it is noted at all. We refer to all these processes jointly as postretrieval monitoring, but it is perhaps worth bearing in mind that such a term is most likely a simplification, as monitoring and retrieval can often proceed in parallel. For example, in relation to monitoring of specific contents of memories, Goldsmith (2016) presented a more sophisticated version of the original framework of Koriat and Goldsmith (1996), in which monitoring and retrieval preceding decisions to volunteer or withhold responses to memory queries are intertwined, proceeding in loops in which retrieval feeds into monitoring, but the output of monitoring also serves to modify retrieval in the next step of this dynamic process. It seems possible that meta-awareness and retrieval from memory can also remain to some extent intertwined, interacting with each other, when more retrieved information increases meta-awareness, but increased metaawareness serves either to expand retrieval or, given that spontaneous memories are not always welcome when one is engaged in other activities, to curtail retrieval and reallocate full attention to the focal task.

The parallel between meta-awareness - as applied to IAMs - and other forms of monitoring of memory is not full, because meta-awareness is unique in its focus not on the contents of memories but rather on their presence. However, just as other forms of monitoring, meta-awareness clearly determines which memories become ultimately reported, independently of any effects on actual retrieval. One can retrieve a large number of memories in cognitive tasks designed to investigate IAMs, but unless one is able to realize that those retrievals occurred, these IAMs cannot be included in any type of report. Meta-awareness thus is a very fundamental form of monitoring that serves as a precondition for other types of analysis of retrieved information, including the analysis of its content. Interestingly, the present study suggests that some manipulations may affect monitoring of the presence of retrievals but not their content-in Experiment 2, the attentional load manipulation affected the meta-awareness assessments but had no discernable effect on the ratings of 
phenomenological qualities of the spontaneously retrieved memory. This dissociation serves to underscore the importance of distinguishing meta-awareness as a separate form of postretrieval monitoring, and it also opens a new avenue of research into how different forms of monitoring relate to each other

The negative effects of attentional load on the rates of IAMs observed here could help clarify the results of a recent study on IAMs in which the effect of cue frequency on the rate of IAMs was observed (Vannucci et al., 2015). In this study, which also used the vigilance task employed here, cue frequency was experimentally manipulated, so that participants were presented with frequent or infrequent verbal cues, with the remaining trials in the vigilance task either left empty or filled with arithmetic operations. It was found that, compared with infrequent cues, both conditions with frequent cues and infrequent cues plus arithmetic operations decreased the number of IAMs reported (for a similar effect on involuntary musical imagery, see Floridou, Williamson, \& Stewart, 2017). The present study suggests that this difference in the incidence of IAMs arose because increased external stimulation - either in the form of frequent verbal cues or arithmetic operations - constituted additional cognitive load that interfered both with retrieval of IAMs and with participants' subjective insight into the contents of their own minds, which is necessary for reporting an IAM.

Our manipulation of attentional load strongly affected the number of IAMs with no significant effects on their phenomenological characteristics, namely, specificity, vividness, pleasantness, and intensity of the feeling experienced during the retrieval. Previous studies investigating the similarities and differences between voluntary and involuntary autobiographical memories have shown that IAMs are more vivid, specific, associated with more intense and immediate emotional reaction at retrieval, and have more impact on mood than their voluntary counterparts (e.g., Berntsen \& Hall, 2004; Rubin, Boals, \& Berntsen, 2008; Schlagman \& Kvavilashvili, 2008; Vannucci et al., 2016). Our null findings suggest that these characteristics of IAMs are largely unaffected by the way attentional load influences involuntary retrieval and postretrieval processes. However, this result needs to be replicated, and future studies should also extend the investigation to other characteristics of memories, such as the importance of memory to the person's life story and identity and the frequency of rehearsal (i.e., thinking and talking) that represent important elements in autobiographical memory.

Apart from informing research on IAMs, the present results are of consequence also for investigations of other forms of spontaneous cognition. One notable example is the case of so called earworms (Beaman \& Williams, 2010), which are experiences of a song playing repeatedly in one's head. Just as IAMs, earworms involve involuntary retrieval of information one experienced earlier, which is often triggered by environmental cues (Williamson et al., 2012). Also, examinations of earworms using the diary method revealed that they often occur in situations of low cognitive load, during activities that do not require much attention (Floridou \& Müllensiefen, 2015), although laboratory-based studies on this phenomenon showed that incidence of earworms can also be increased when a more difficult task is administered (Hyman et al., 2013) - a finding potentially underscoring the need to distinguish between task engagement and task difficulty, as discussed earlier.

The similarities across different manifestations of spontaneous cognition point to the urgent need for developing a comprehensive framework of such phenomena. The most advanced theorizing on this topic has so far been developed within the literature on mind wandering, although for a long time the lines of research focused on IAMs and mind wandering have developed to a large extent independently. Only recently increasing attention has been devoted to the discussion of the theoretical links between the two phenomena (McVay \& Kane, 2013; Plimpton, Patel, \& Kvavilashvili, 2015; Rasmussen \& Berntsen, 2009, 2011; Vannucci, Pelagatti, \& Marchetti, 2017).

Both IAMs and the episodes of mind wandering refer to mental contents that are incidental to the focal task and spontaneously generated (but see Seli, Risko, Smilek, \& Schacter, 2016, for a discussion of intentional mind wandering), potentially creating a common conceptual framework encompassing both phenomena. Indeed, a significant proportion of episodes examined in mind wandering studies have been described as concerning personal past and thus could be possibly classified as IAMs (Smallwood, Nind, \& O'Connor, 2009; Stawarczyk, Cassol, \& D'Argembeau, 2013). However, the theoretical considerations of IAMs and mind wandering differ in one important aspect. IAMs are clearly cue dependent (Berntsen, 1996; Berntsen \& Hall, 2004), with a number of studies being devoted specifically to elucidating the nature of the cues that are most likely to elicit IAMs. Indeed, the cue-dependent nature of IAMs forms the basis of the procedures used to investigate this phenomenon, including the procedure used in the present study. By contrast, in the mind-wandering literature, mind-wandering episodes have been mainly described as self-generated (e.g., Smallwood, 2013) and stimulus independent (Antrobus, 1968), terms that emphasize their independence from perception and ongoing actions. Interestingly, over the last few years, an increasing number of studies have shown that environmental stimuli indeed trigger mind wandering episodes (Maillet \& Schacter, 2016; Maillet, Seli, \& Schacter, 2017; McVay \& Kane, 2013; Plimpton et al., 2015; Song \& Wang, 2012; Vannucci et al., 2017).

Previous studies on mind wandering have consistently shown that the frequency of mind wandering depends heavily on the cognitive demands of the ongoing task. The rate of 
mind wandering is reduced whenever the focal task is made more difficult, requiring a stronger involvement of the attentional processes operating within the working memory system (Levinson, Smallwood, \& Davidson, 2012; Teasdale et al., 1995) or focused attention (e.g., manipulation of perceptual load; Forster \& Lavie, 2009) - that is, when the attentional load is increased. Our results on the effects of attentional load on retrieval and postretrieval processes of IAMs, collected within a paradigm designed specifically to assess IAMs, clearly parallel the observations reported in the mind-wandering studies and add to the argument for common cognitive principles governing mind wandering and IAMs.

Apart from exploring the links between mind wandering and IAMs, three other future directions present themselves. First, in Experiment 1 we found that for IAMs reported as being triggered by word phrases, retrieval times (RTs) were slower in the High-AL group compared with the Low-AL group. These findings suggest that attentional load might also interfere with the time needed to generate and/or become aware of an IAM. So far, research on IAMs has been focused on the rates of IAMs but these results suggest that the temporal dynamics of IAMs should also be explored.

Second, in the present study we investigated the effects of attentional load on IAMs and their meta-awareness in a sample of young adults. Future studies might investigate these effects in other populations of special interest for research on IAMs, such as elderly people. Several studies investigating IAMs across the life span using diary methods (Schlagman, Kliegel, Schulz, \& Kvavilashvili, 2009; Schlagman, Kvavilashvili, \& Schulz, 2007) found small but significant differences between IAMs of young and older adults, with an age-related reduction in IAMs when elderly participants reported being more concentrated on the ongoing activities compared with younger adults. However, other studies using retrospective questionnaires (Berntsen \& Rubin, 2002; Berntsen, Rubin, \& Salgado, 2015; Moulin et al., 2014) reported no age-related differences. In a very recent line of studies, Berntsen, Rasmussen, Miles, Nielsen, and Ramsgaard (2017) examined involuntary memories in young and older adults in a laboratory setting (i.e., involuntary memories of a film of a simulated event) and during a normal day in their life. Across both studies, no significant age differences in the frequency of involuntary memories were found. We suggest that future studies are needed to reconcile these discrepancies, and such studies could build on our results revealing the role of attentional load in governing IAMs by investigating the association between age-related changes in attentional abilities and involuntary retrieval of autobiographical memories.

Finally, in our study we focused on ordinary, daily-life IAMs. However, research on spontaneously evoked memories is of importance not only for understanding cognition in standard, everyday situations but also for advancing investigations into abnormal forms of cognition, such as intrusive memories occurring in posttraumatic stress disorder. For this reason, future studies might extend the investigation of the role of attentional load in governing spontaneous memories to unpleasant and unwanted intrusive memories/images for negative or adverse material. Recent studies on trauma-related intrusions reported that people are not always aware of them (i.e., people may lack meta-awareness of their trauma-related thoughts; Takarangi et al., 2015; Takarangi et al., 2017; Takarangi et al., 2014), suggesting that self-report may underestimate the frequency of intrusive thoughts and memories. Future studies are needed to investigate whether and how (i.e., retrieval and postretrieval processes) attentional load might affect the frequency of occurrence of this other kind of spontaneous cognition.

Acknowledgements The authors would like to thank Dr. Bruno Bocanegra for helpful and thoughtful discussions and advice on the design of Experiment 1. We also would like to thank Martina Fioravanti for her help with data collection.

\section{Compliance with ethical standards}

Conflict of interest All the authors declare that they have no conflict of interest.

All procedures performed in the study are in accordance with the ethical standards of the institutional research committee and with the 1964 Helsinki declaration and its later amendments or comparable ethical standards.

Informed consent Informed consent was obtained from all individual participants included in the study.

\section{References}

Anderson, M. C., \& Spellman, B. A. (1995). On the status of inhibitory mechanisms in cognition: Memory retrieval as a model case. Psychological Review, 102(1), 68-100. doi:https://doi.org/10.1037/ 0033-295X.102.1.68

Antrobus, J. S. (1968). Information theory and stimulus-independent thought. British Journal of Psychology, 59(4), 423-430. doi: https://doi.org/10.1111/j.2044-8295.1968.tb01157.x

Asendorpf, J. B., Conner, M., De Fruyt, F., De Houwer, J., Denissen, J. J. A., Fiedler, K., . . . Wicherts, J. M. (2013). Recommendations for increasing replicability in psychology. European Journal of Personality, 27(2), 108-119. doi:https://doi.org/10.1002/per.1919

Baddeley, A. (1993). Short-term phonological memory and long-term learning: A single case study. European Journal of Cognitive Psychology, 5(2), 129-148. doi:https://doi.org/10.1080/ 09541449308520112

Ball, C. T. (2007). Can we elicit involuntary autobiographical memories in the laboratory? In J. H. Mace (Ed.), Involuntary memory (pp. 127-152). Oxford, UK: Blackwell. doi:https://doi.org/10.1002/ 9780470774069.ch7

Barzykowski, K., \& Niedźwieńska, A. (2016). The effects of instruction on the frequency and characteristics of involuntary autobiographical memories. PLOS ONE, 11(6), e0157121. doi:https://doi.org/10. 1371/journal.pone.0157121

Barzykowski, K., \& Niedźwieńska, A. (2018). Involuntary autobiographical memories are relatively more often reported during high 
cognitive load tasks. Acta Psychologica, 182, 119-128. doi:https:// doi.org/10.1016/j.actpsy.2017.11.014

Beaman, C. P., \& Williams, T. I. (2010). Earworms ('stuck song syndrome'): Towards a natural history of intrusive thoughts. British Journal of Psychology, 101, 637-653. doi:https://doi.org/10.1348/ 000712609X479636

Berntsen, D. (1996). Involuntary autobiographical memories. Applied Cognitive Psychology, 10(5), 435-454. doi:https://doi.org/10.1002/ (SICI)1099-0720(199610)10:5<435::AID-ACP408>3.0.CO;2-L

Berntsen, D. (2010). The unbidden past: Involuntary autobiographical memories as a basic mode of remembering. Current Directions in Psychological Science, 19(3), 138-142. doi:https://doi.org/10.1177/ 0963721410370301

Berntsen, D., \& Hall, N. M. (2004). The episodic nature of involuntary autobiographical memories. Memory \& Cognition, 32(5), 789-803. doi:https://doi.org/10.3758/BF03195869

Berntsen, D., Rasmussen, A. S., Miles, A. N., Nielsen, N. P., \& Ramsgaard, S. B. (2017). Spontaneous or intentional? Involuntary versus voluntary episodic memories in older and younger adults. Psychology and Aging, 32(2), 192-201. doi:https://doi.org/10. 1037/pag0000157

Berntsen, D., \& Rubin, D. C. (2002). Emotionally charged autobiographical memories across the life span: The recall of happy, sad, traumatic, and involuntary memories. Psychology and Aging, 17(4), 636-652. doi:https://doi.org/10.1037/0882-7974.17.4.636

Berntsen, D., Rubin, D. C., \& Salgado, S. (2015). The frequency of involuntary autobiographical memories and future thoughts in relation to daydreaming, emotional distress, and age. Consciousness and Cognition, 36, 352-372. doi:https://doi.org/10.1016/j.concog. 2015.07.007

Berntsen, D., Staugaard, S. R., \& Sørensen, L. M. T. (2013). Why am I remembering this now? Predicting the occurrence of involuntary (spontaneous) episodic memories. Journal of Experimental Psychology: General, 142(2), 426-444. doi:https://doi.org/10. 1037/a0029128

Conway, M. A., \& Pleydell-Pearce, C. W. (2000). The construction of autobiographical memories in the self-memory system. Psychological Review, 107, 261-288.

Craik, F. I. M., Govoni, R., Naveh-Benjamin, M., \& Anderson, N. D. (1996). The effects of divided attention on encoding and retrieval processes in human memory. Journal of Experimental Psychology: General, 125, 159-180.

Evans, L. H., Williams, A. N., \& Wilding, E. L. (2015). Electrophysiological evidence for retrieval mode immediately after a task switch. NeuroImage, 108, 435-440. doi:https://doi.org/10. 1016/j.neuroimage.2014.12.068

Faul, F., Erdfelder, E., Lang, A.-G., \& Buchner, A. (2007). G*Power 3: A Flexible statistical power analysis program for the social, behavioral, and biomedical sciences. Behavioral Research Methods, 39(2), 175-191. doi:https://doi.org/10.3758/BF03193146

Floridou, G. A., \& Müllensiefen, D. (2015). Environmental and mental conditions predicting the experience of involuntary musical imagery: An experience sampling method study. Consciousness and Cognition, 33, 472-486. doi:https://doi.org/10.1016/j.concog.2015. 02.012

Floridou, G. A., Williamson, V. J., \& Stewart, L. (2017). A novel indirect method for capturing involuntary musical imagery under varying cognitive load. The Quarterly Journal of Experimental Psychology, 70(11), 2189-2199. doi:https://doi.org/10.1080/ 17470218.2016 .1227860

Forster, S., \& Lavie, N. (2009). Harnessing the wandering mind: The role of perceptual load. Cognition, 111(3), 345-355. doi:https://doi.org/ 10.1016/j.cognition.2009.02.006

Funder, D. C., Levine, J. M., Mackie, D. M., Morf, C. C., Sansone, C., Vazire, S., \& West, S. G. (2014). Improving the dependability of research in personality and social psychology: Recommendations for research and educational practice. Personality and Social Psychology Review, 18(1), 3-12. doi:https://doi.org/10.1177/ 1088868313507536

Goldsmith, M. (2016). Metacognitive quality-control processes in memory retrieval and reporting. In J. Dunlosky \& S. K. Tauber (Eds.), Oxford library of psychology: The Oxford handbook of metamemory (pp. 357-385). New York, NY: Oxford University Press.

Goldsmith, M., Koriat, K., \& Weinberg-Eliezer, A. (2002). Strategic regulation of grain size memory reporting. Journal of Experimental Psychology: General, 131, 73-95. doi:https://doi.org/10.1037/ 0096-3445.131.1.73

Hanczakowski, M., Pasek, T., Zawadzka, K., \& Mazzoni, G. (2013). Cue familiarity and 'don't know' responding in episodic memory tasks. Journal of Memory and Language, 69, 368-383. doi:https://doi.org/ 10.1016/j.jml.2013.04.005

Herron, J. E., Evans, L. H., \& Wilding, E. L. (2016). Electrophysiological evidence for flexible goal-directed cue processing during episodic retrieval. NeuroImage, 132, 24-31. doi:https://doi.org/10.1016/j. neuroimage.2016.02.025

Herron, J. E., \& Rugg, M. D. (2003). Retrieval orientation and the control of recollection. Journal of Cognitive Neuroscience, 15, 842-854. doi:https://doi.org/10.1162/089892903322370762

Hyman, I. E., Burland, N. K., Duskin, H. M., Cook, M. C., Roy, C. M., McGrath, J. C., \& Roundhill, R. F. (2013). Going gaga: Investigating, creating, and manipulating the song stuck in my head. Applied Cognitive Psychology, 27, 204-215. doi:https://doi.org/10. 1002/acp.2897

Jahng, S., Wood, P. K., \& Trull, T. J. (2008). Analysis of affective instability in ecological momentary assessment: Indices using successive difference and group comparison via multilevel modeling. Psychological Methods, 13(4), 354-375. doi:https://doi.org/10. 1037/a0014173

Koriat, A., \& Goldsmith, M. (1996). Monitoring and control processes in the strategic regulation of memory accuracy. Psychological Review, 103(3), 490-517. doi:https://doi.org/10.1037//0033-295X.103.3. 490

Kvavilashvili, L., \& Mandler, G. (2004). Out of one's mind: A study of involuntary semantic memories. Cognitive Psychology, 48(1), 4794. doi:https://doi.org/10.1016/S0010-0285(03)00115-4

Levinson, D. B., Smallwood, J., \& Davidson, R. (2012). The persistence of thought: Evidence for a role of working memory in the maintenance of task-unrelated thinking. Psychological Science, 23, 375380. doi:https://doi.org/10.1177/0956797611431465

Mace, J. H. (2004). Involuntary autobiographical memories are highly dependent on abstract cuing: The Proustian view is incorrect. Applied Cognitive Psychology, 18, 893-899. doi:https://doi.org/10. 1002/acp. 1020

Mace, J. H. (2007). Involuntary memory. Oxford, UK: Blackwell. doi: https://doi.org/10.1002/9780470774069.fmatter

Maillet, D., \& Schacter, D. L. (2016). When the mind wanders: Distinguishing stimulus-dependent from stimulus-independent thoughts during incidental encoding in young and older adults. Psychology and Aging, 31(4), 370-379. doi:https://doi.org/10. 1037/pag0000099

Maillet, D., Seli, P., \& Schacter, D. L. (2017). Mind-wandering and task stimuli: Stimulus-dependent thoughts influence performance on memory tasks and are more often past- versus future-oriented. Consciousness and Cognition, 52, 55-67. doi:https://doi.org/10. 1016/j.concog.2017.04.014

Mandler, G. (1994). Hypermnesia, incubation, and mind popping: On remembering without really trying. In C. Umiltà \& M. Moscovitch (Eds.), Attention and performance XV (pp. 3-33). Cambridge, MA: MIT Press.

Mazzoni, G., Vannucci, M., \& Batool, I. (2014). Manipulating cues in involuntary autobiographical memory: Verbal cues are more 
effective than pictorial cues. Memory \& Cognition, 42(7), 10761085. doi:https://doi.org/10.3758/s13421-014-0420-3

McVay, J. C., \& Kane, M. J. (2013). Dispatching the wandering mind? Toward a laboratory method for cuing "spontaneous" off-task thought. Frontiers in Psychology, 4, 570. doi:https://doi.org/10. 3389/fpsyg.2013.00570

Moulin, C. J. A., Souchay, C., Buchanan, S., Bradley, R., Karadoller, D. Z., \& Akan, M. (2014). Déjà vu in older adults. In B. L. Schwartz \& A. S. Brown (Eds.), Tip-of-the-tongue states and related phenomena (pp. 281-304). New York, NY: Cambridge University Press.

Plimpton, B., Patel, P., \& Kvavilashvili, L. (2015). Role of triggers and dysphoria in mind-wandering about past, present and future: A laboratory study. Consciousness and Cognition, 33, 261-276. doi: https://doi.org/10.1016/j.concog.2015.01.014

Poh, J. H., Chong, P. L., \& Chee, M. W. (2016). Sleepless night, restless mind: Effects of sleep deprivation on mind wandering. Journal of Experimental Psychology: General, 145(10), 1312-1318. doi: https://doi.org/10.1037/xge0000207

Rasmussen, A. S., \& Berntsen, D. (2009). The possible functions of involuntary autobiographical memories. Applied Cognitive Psychology, 23(8), 1137-1152. doi:https://doi.org/10.1002/acp. 1615

Rasmussen, A. S., \& Berntsen, D. (2011). The unpredictable past: Spontaneous autobiographical memories outnumber autobiographical memories retrieved strategically. Consciousness and Cognition, 20(4), 1842-1846. doi:https://doi.org/10.1016/j.concog.2011.07. 010

Rubin, D. C. (1995). Memory in oral traditions: The cognitive psychology of epic, ballads, and counting-out rhymes. New York, NY: Oxford University Press.

Rubin, D. C., Boals, A., \& Berntsen, D. (2008). Memory in posttraumatic stress disorder: Properties of voluntary and involuntary, traumatic and nontraumatic autobiographical memories in people with and without posttraumatic stress disorder symptoms. Journal of Experimental Psychology: General, 137, 591-614. doi:https://doi. org/10.1037/a0013165

Schlagman, S., Kliegel, M., Schulz, J., \& Kvavilashvili, L. (2009). Differential effects of age on involuntary and voluntary autobiographical memory. Psychology and Aging, 24(2), 397-411. doi: https://doi.org/10.1037/a0015785

Schlagman, S., \& Kvavilashvili, L. (2008). Involuntary autobiographical memories in and outside the laboratory: How different are they from voluntary autobiographical memories? Memory \& Cognition, 36(5), 920-932. doi:https://doi.org/10.3758/MC.36.5.920

Schlagman, S., Kvavilashvili, L., \& Schulz, J. (2007). Effects of age on involuntary autobiographical memories. In J. H. Mace (Ed.), Involuntary memory (pp. 87-112). Oxford, UK: Blackwell. doi: https://doi.org/10.1002/9780470774069.ch5

Schooler, J. W. (2002). Re-representing consciousness: Dissociations between consciousness and meta-consciousness. Trends in Cognitive Sciences, 6(8), 339-344. doi:https://doi.org/10.1016/S13646613(02)01949-6

Schooler, J. W., Smallwood, J., Christoff, K., Handy, T. C., Reichle, E. D., \& Sayette, M. A. (2011). Meta-awareness, perceptual decoupling and the wandering mind. Trends in Cognitive Sciences, 15(7), 319-326. doi:https://doi.org/10.1016/j.tics.2011.05.006

Seli, P., Risko, E. F., Smilek, D., \& Schacter, D. L. (2016). Mindwandering with and without intention. Trends in Cognitive Sciences, 20(8), 605-617. doi:https://doi.org/10.1016/j.tics.2016. 05.010

Smallwood, J. (2013). Distinguishing how from why the mind wanders: A process-occurrence framework for self-generated mental activity. Psychological Bulletin, 139(3), 519-535. doi:https://doi.org/10. 1037/a0030010
Smallwood, J., Nind, L., \& O'Connor, R. C. (2009). When is your head at? An exploration of the factors associated with the temporal focus of the wandering mind. Consciousness and Cognition, 18(1), 118125. doi:https://doi.org/10.1016/j.concog.2008.11.004

Smallwood, J., \& Schooler, J. W. (2015). The science of mind wandering: Empirically navigating the stream of consciousness. Annual Review of Psychology, 66, 487-518. doi:https://doi.org/10.1146/annurevpsych-010814-015331

Song, X., \& Wang, X. (2012). Mind wandering in Chinese daily livesAn experience sampling study. PLOS ONE, 7(9), e44423. doi: https://doi.org/10.1371/journal.pone.0044423

Sörqvist, P., \& Marsh, J. E. (2015). How concentration shields against distraction. Current Directions in Psychological Science, 24, $267-$ 272. doi:https://doi.org/10.1177/0963721415577356

Stawarczyk, D., Cassol, H., \& D'Argembeau, A. (2013). Phenomenology of future-oriented mind-wandering episodes. Frontiers in Psychology, 4, 425. doi:https://doi.org/10.3389/fpsyg.2013.00425

Takarangi, M. K. T., Lindsay, D. S., \& Strange, D. (2015). Metaawareness and the involuntary memory spectrum: Reply to Meyer, Otgaar, and Smeets (2015). Consciousness and Cognition, 34, 1-3. doi:https://doi.org/10.1016/j.concog.2015.03.009

Takarangi, M. K. T., Nayda, D., Strange, D., \& Nixon, R. D. V. (2017). Do meta-cognitive beliefs affect meta-awareness of intrusive thoughts about trauma? Journal of Behavior Therapy and Experimental Psychiatry, 54, 292-300. doi:https://doi.org/10.1016/ j.jbtep.2016.10.005

Takarangi, M. K. T., Strange, D., \& Lindsay, D. S. (2014). Self-report may underestimate trauma intrusions. Consciousness and Cognition, 27, 297-305. doi:https://doi.org/10.1016/j.concog.2014. 06.002

Teasdale, J. D., Dritschel, B. H., Taylor, M. J., Proctor, L., Lloyd, C. A., Nimmo-Smith, I., \& Baddeley, A. D. (1995). Stimulus-independent thought depends on central executive resources. Memory \& Cognition, 23(5), 551-559. doi:https://doi.org/10.3758/ BF03197257

Vannucci, M., Batool, I., Pelagatti, C., \& Mazzoni, G. (2014). Modifying the frequency and characteristics of involuntary autobiographical memories. PLOS ONE, 9(4), e89582. doi:https://doi.org/10.1371/ journal.pone. 0089582

Vannucci, M., Pelagatti, C., Chiorri, C., \& Mazzoni, G. (2016). Visual object imagery and autobiographical memory: Object imagers are better at remembering their personal past. Memory, 24(4), 455-470. doi:https://doi.org/10.1080/09658211.2015.1018277

Vannucci, M., Pelagatti, C., Hanczakowski, M., Mazzoni, G., \& Rossi Paccani, C. (2015). Why are we not flooded by involuntary autobiographical memories? Few cues are more effective than many. Psychological Research, 79(6), 1077-1085. doi:https://doi.org/10. 1007/s00426-014-0632-y

Vannucci, M., Pelagatti, C., \& Marchetti, I. (2017). Manipulating cues in mind wandering: Verbal cues affect the frequency and the temporal focus of mind wandering. Consciousness and Cognition, 53, 61-69. doi:https://doi.org/10.1016/j.concog.2017.06.004

Williamson, V. J., Jilka, S. R., Fry, J., Finkel, S., Müllensiefen, D., \& Stewart, L. (2012). How do "earworms" start? Classifying the everyday circumstances of involuntary musical imagery. Psychology of Music, 40, 259-284. doi:https://doi.org/10.1177/ 0305735611418553

Wixted, J. T., \& Mickes, L. (2010). A continuous dual-process model of remember/know judgments. Psychological Review, 117, 10251054. doi:https://doi.org/10.1037/a0020874

Zawadzka, K., Hanczakowski, M., \& Wilding, E. L. (2017). Late consequences of early selection: When memory monitoring backfires. Journal of Memory and Language, 92, 114-127. doi:https://doi. org/10.1016/j.jml.2016.06.003 